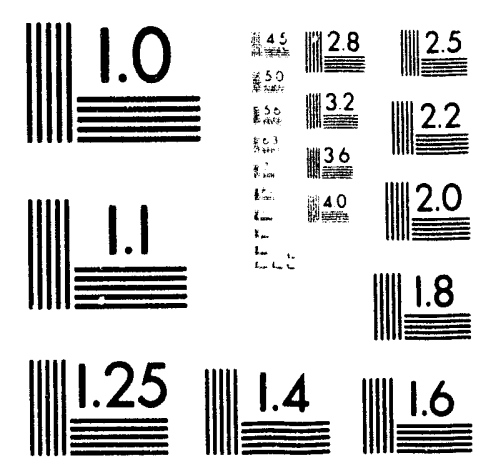



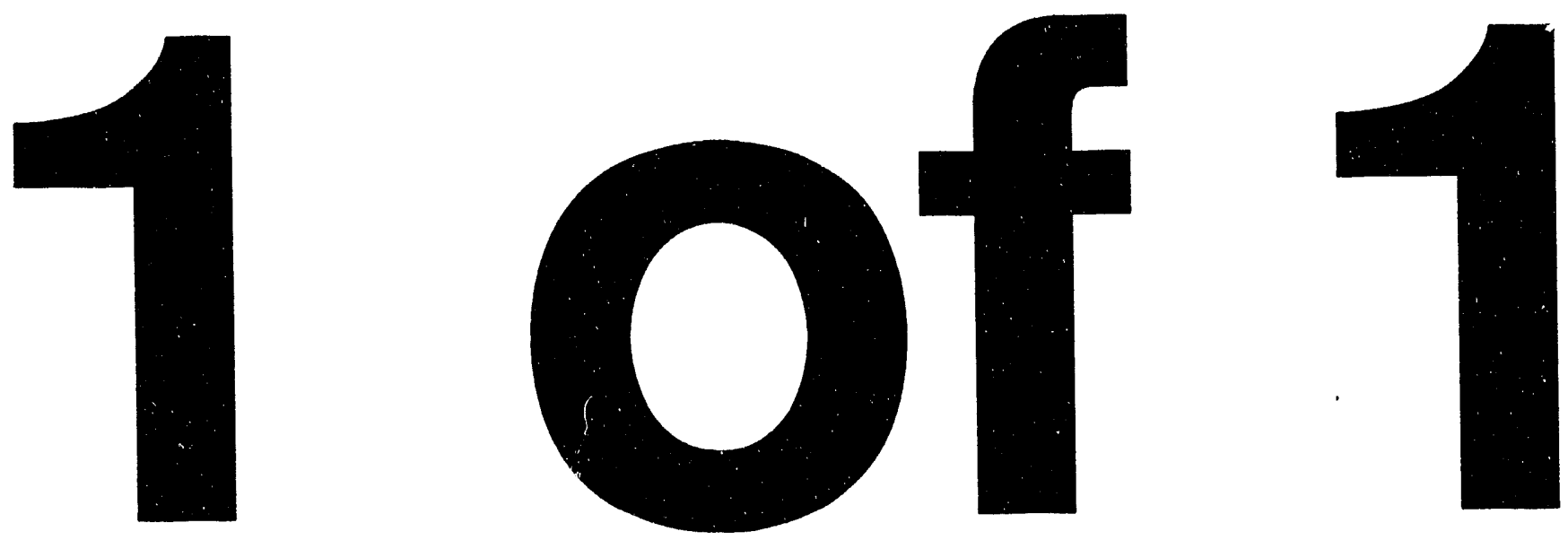
LBL-33220

UC-410

LSGN-115

\title{
SURVEY AND ALIGNMENT DATA ANALYSIS FOR THE ALS STORAGE RING
}

\author{
RODERICH KELLER \\ ACCELERATOR and FUSION RESEARCH DIVISION \\ Lawrence Berkeley Laboratory \\ University of California \\ Berkeley, CA 94720
}

MAY 1993

\section{MASTER}

This work was supported by the Director, Office of Energy Research, Office of Basic Energy Sciences, Materials Sciences Division, of the U.S. Department of Energy under Contract No. DE-AC03-76SF00098. 


\title{
Survey and Alignment Data Analysis for the ALS Storage Ring*
}

\author{
Roderich Keller \\ Lawrence Berkeley Laboratory \\ University of California, Berkeley, CA 94720 USA
}

\section{Abstract}

The survey and alignment effort for the Advanced Light Source (ALS) accelerator complex has been described elsewhere[1]. Data analysis for this task comprises the creation of ideal data, comparison of measured coordinates with ideal ones, and computation of alignment values, taking into account the effects caused by finite observation accuracy. A novel approach has been taken, using personal computer spreadsheets rather than more conventional programming methods. This approach was induced by the necessities to create and frequently refine the analysis procedures while measurements were already underway, and further by hardware constraints that limited the use of an available surveying code. A major benefit consists in the ability to identify and deal with discrepancies that occasionally arise when different techniques are used to observe the same object, in a timely and efficient manner. As a result of the performed survey and alignment work, the ALS lattice magnets have been positioned with accuracies well exceeding the original specifications.

\section{INTRODUCTION}

The Advanced Light Source (ALS) electron storage ring, now being commissioned at Lawrence Berkeley Laboratory, is the main accelerator of a third-generation synchrotron radiation source designed to produce extremely bright photon beams in the UVand soft X-ray regions [2]. The 1-1.9-GeV ring consists of 12 superperiods with $196.8 \mathrm{~m}$ total circumference and has particularly tight positioning tolerances for lattice magnets and other components to assure the required characteristics.
The general survey and alignment concept for the ALS[1] is based on a neiwork of fixed monuments installed in the building floor, to which all component positions are referred. Contrary to the original intent, the commercial software package ECDS ${ }^{\circledR}$ is being used for data acquisition, bundling, and transformations from observation station into object coordinate systems. Theodolites only are used as observation instruments with ECDS, and an absolute scale has to be established by observing some object of precisely known length.

For the tasks of creating ideal data and computing alignment values, spreadsheets were developed by the author using the application EXCEL ${ }^{\circledR}$ for Macintosh ${ }^{\circledR}$ computers. Choice of a spreadsheet method rather than conventional programming techniques proved very convenient when in the course of this work the sheets had to he progressively modified under severe time pressure to include new effects and help redefine the observation procedures. With spreadsheets, varying input data formats coming from the survey crew could be easily accommodated, and adding numerous consistency checks as well as generating additional ideal data for special alignment tasks was possible without much effort. Dedicated spreadsheets were created for each of the 12 curved sectors of the storage ring.

In this paper, the features of these spreadsheets are presented, and the obtained alignment results for lattice and corrector magnets are discussed.

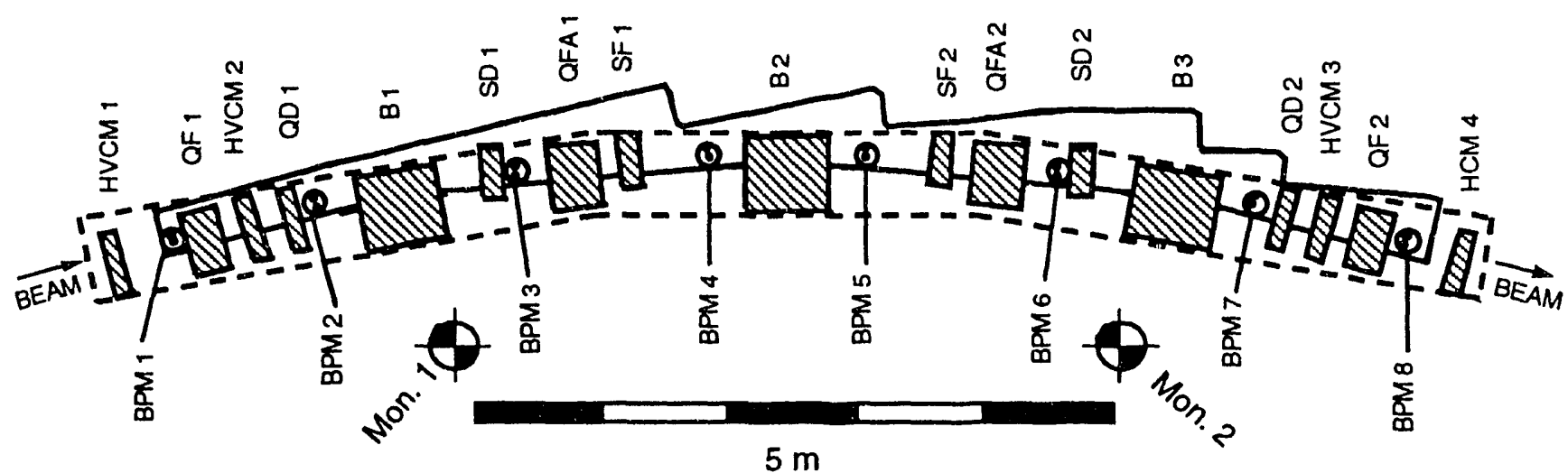

Figure 1. Storage ring magnet lattice in one curved section, with outlines of vacuum chamber (solid line) and girder (broken line). For magnet designations, see Table 1. Four monuments, Mon., are used as survey references; two of them, near the adjacent straight section centers, are not shown in this figure.

\begin{abstract}
*Work supported by the Director, Office of Energy Research, Office of Basic Energy Sciences, Materials Sciences Division U.S. Department of Energy, under Contract No. DE-AC03-76SF00098.
\end{abstract}

\section{SCOPE AND TOLERANCES}

Storage ring objects designated for precision alignment include: a), lattice magnets ( 36 bend magnets, 72 quadrupoles, and 48 sextupoles); b), 46 corrector magnets; 
c), special magnets ( 2 septa and 4 bump magnets); d) 12 storage ring vacuum chambers, represented by 96 beam position monitors (BPM), 8 per chamber; e), 2 rf cavities; and $f$ ), special objects (photon beamline components and gate valves). A list of required local tolerances for objects discussed in this paper is given in Table 1. These values are understood as 1- $\sigma$ half-widths of every error distribution, with a $2-\sigma$ cutoff. No strict global tolerance value is established.

Every magnet carries four fiducial posts that are welded to its upper side without attempting to achieve any precise positioning; the positions are determined by a coordinate measurement machine. Different exchangeable targets are used on these posts, either optical targets with engraved circle and center point for surveying or tooling balls, for alignment in combination with dial indicators. The BPMs can be equipped with one target, each.

Table 1

Local Alignment Tolerances

\begin{tabular}{|c|c|c|c|c|c|c|}
\hline Object & $\begin{array}{c}\Delta \mathrm{w} \\
{[\mathrm{mm}]}\end{array}$ & $\underset{[\mathrm{mm}]}{\Delta \mathrm{u}}$ & $\begin{array}{c}\Delta \mathrm{v} \\
{[\mathrm{mm}]}\end{array}$ & $\begin{array}{c}\Delta \mathbf{u}^{\prime} \\
{[\mathrm{mrad}]}\end{array}$ & $\begin{array}{c}\Delta v^{\prime} \\
{[\mathrm{mrad}]}\end{array}$ & $\begin{array}{c}\Delta \mathrm{w}^{\prime} \\
{[\mathrm{mrad}]}\end{array}$ \\
\hline B & 0.15 & 0.15 & 0.15 & J. & 1 & 0.25 \\
\hline QD & 0.3 & 0.15 & 0.15 & J. & J. & 0.5 \\
\hline $\mathrm{QF}$ & 0.3 & 0.15 & 0.15 & J. & $J$. & 0.5 \\
\hline QFA & 0.3 & 0.15 & 0.15 & J. & J. & 0.5 \\
\hline SF & 0.5 & 0.15 & 0.15 & J. & J. & J. \\
\hline SD & 0.5 & 0.15 & 0.15 & J. & 1 & J. \\
\hline HVC & 1.0 & 1.0 & 1.0 & J. & $J$ & 2.0 \\
\hline BPM & 0.15 & 0.15 & 0.15 & 1. & 1 & $J$ \\
\hline
\end{tabular}

$B$, bend magnet. $Q D$, defocusing quadrupole. $Q F$ and QFA, focusing quadrupoles. SF, focusing sextupole. SD, defocusing sextupole. HVC, horizontal and vertical corrector magnet. BPM, beam-position monitor. Tolerances are described in local, beam-following coordinates: $w$, in beam direction; $u$, radially away from the ring center; $v$, vertically up. u', pitch; v', yaw; w'. roll. ./. indicates that these values are predetermined by other values in this table.

\section{INSTALLATION AND ALIGNMENT}

Magnets and vacuum chambers are mounted on girders spanning one of the twelve curved storage-ring sectors each, see Figure 1. In the first phase of installation, the vacuum chambers are precisely aligned to their girders, represented by 12 girder fiducials. Then the magnets are installed and fine aligned to the girders as well. As a last step, girders and their objects are aligned to the global 'ALS Coordinate System' represented by the four floor monuments in the immediate neighborhood of every girder. This scheme led to the creation of two families of spreadsheets in which objectalignment data were computed, the so-called 'girder' and 'monument' sheets.

This concept of separation between local alignment of objects (magnets or chambers) to girder fiducials and final global alignment of girders to monuments is well suited in principle to minimize the entire effort, but in reality it failed because the girders bent too much under the weight of the installed magnets, and thus the original girder fiducialization was lost. Therefore, some magnets had to be fine- aligned to girders using survey data related to the global monument sytem, and the final alignment of the vacuum chambers was performed using optical tooling methods since most chamber fiducials are hidden by the magnets themselves.

All alignment values are ultimately expressed in local, beam-following coordinates along the main object axes 10 facilitate the orientation of dial indicators with which the alignment is controlled on every fiducial. Even the alignment to monuments, executed by moving girders only, is monitored on selected magnet fiducials, to achieve final alignment of lattice magnets to the global ALS coordinate system in the most direct manner.

\section{CREATION OF IDEAL DATA}

Ideal data for all objects included in the survey and alignment effort are created in another line of spreadsheets, based on mechanical fiducialization data [3] and magnetic measurements [4]. Special effects are accounted for, such as magnetic-to-mechanical axis offsets for quadrupoles and sextupoles, and roll angles introduced by shimming the two halves of every quadrupole.

These data, together with an accelerator lattice file [5] and the information on the lattice position of any given magnet in the entire ring are being used to convert all fiducial data from local magnet systems into a generic girder system whose origin horizontally coincides with the intersection of the tangents to the ideal beam trajectcry on both sides of the central bend magnet. The tra.sformation into the global ALS system is done inside the 'monument sheets,' individually for every storage ring sector.

\section{GIRDER SHEET}

The Girder Sheet is the first of the tivo spreadsheet varieties in which ideal and observed magnet fiducial positions are compared and then adjusted correction values are computed. As a first step, the ideal magnet fiducial data are modified to account for the measured offset of the vacuum chamber center against the ideal girder system. The chambers cannot be moved in this area because they are anchored on a pinned stanchion. After calculating the idealto-observed position differences in all three directions of this modified girder system, the longitudinal difference values are compensated for thermal expansion of the girder at the time of surveying. Observed lateral girder deformations, caused by their inner support structures, ultimately made it necessary to maintain the entire storage ring tunnel at the design temperature $\left(23.9 \pm 1{ }^{\circ} \mathrm{C}\right)$ during surveys, but the longitudinal compensation algorithm is kept in the girder sheets as a safeguard against larger actual temperature excursions.

Two kinds of consistency checks are permanently included in the Girder Sheets, a comparison of the distances between all fiducials of one magnet from both optical and mechanical measurements, and a comparison of all distances between the four observed monuments with the same values derived from the latest dedicated monument survey. These latter ("ideal") monument distances are included as constants in the ECDS data file to provide absolute scaling. 
To facilitate an easy set-up of the dial indicators the derived magnet correction values are transformed from the common girder coordinate system into individual magnet systems. Because of the redundancy of information provided by 12 coordinate values some adjustments are made before the correction values are finalized. The precision of the original instailation, compared to magnei dimensions, allows one to compute the adjusted corrections sequentially, rather than as a true rigid-body movement. For every magnet, the average shifts along its nwn major axes are calculated first, and the remaining correction values are used to evaluate average yaw, pitch, and roll angles which are then translated back into fiducial shifts along the magnet axes, to be superimposed on the average shifts.

\section{MONUMENT SHEET}

The Monument Sheet computes the final alignment values for all magnets, to be executed by moving entire girders only. This implies that all magnet correction values are averaged to yield global girder corrections, i.e. average shifts in three directions and three angular rotations. These global corrections, however, are then expressed as shift values for fiducials on the two focusing quadrupoles at the end of every girder (horizontal directions) and on the central bend magnet in the girder center (vertical direction). Munument Sheets include the same consistency checks as Girder Sheets, but their input and ideal data are expressed in the global ALS coordinate system. One given set of observation data can be easily transformed by ECDS into both ALS and individual girder systems.

After the initial calculation of ideal-to-observed fiducial position differences in the ALS system, these values are transformed into the conesponding girder system, and the temperature compensation algorithm is applied. At this point there is the option to merge individual magnet corrections, resulting from executing the values obtained from the Girder Sheet, into the Monument Sheet, reducing its remaining corrections accordingly. The average shifts for all magnets are calculated next, and from the remaining correction values the three angular corrections are detcrmined. For pitch and yaw, only magnets at the ends of the girders, including the outer bend magnets, are taken into account, and for roll, only those fiducials are taken that are more than $2.50 \mathrm{~mm}$ away from the girder mid-line. The evaluated ingles are then used for a second-order correction, assuring that the average shift for all fiducials resulting from the angular corr ctions is exactly zero. In addition, the differences between all individual corrections and the effects of the computed global corrections on every magnet fiducial are displayed for visual inspection. All fiducial corrections resulting from the global girder corrections are transformed into local magnet coordinate systems, but the reference fiducial corrections to be used to monitor the girder alignment are together displayed again, for the alignment technicians' use.

\section{RESULTS}

Ideally, 3 surveys and 2 alignments are the minimum number, but due to girder deformation under temperature changes and mechanical load, changes of the scaling reference, ground motion, and bakeout of two vacuum chambers, it took 7.7 surveys on the average to reach good alignment for all magnets. Two gisders actually needed three surveys only, each. The final lattice magnet alignment exceeded the requirements by far. As an iliustration, Iwo sets of data are presented in Tables 2 and 3. They represent standard deviations of the remaining position errors for all lattice magnets in absolute. Table 2 , and after subtracting a linear fitting line separately for every girder in the transverse coordinates, and the average error in the longitudinal coordinate, Table 3 . Roll errors are given as absolute standard deviations, Table 2 , and as averages of absolute errors, Table 3.

Table 2

Final Absolute Alignment Enors

\begin{tabular}{|c|c|c|c|c|}
\hline & $\mathrm{dw}[\mathrm{mm}]$ & $\mathrm{du}[\mathrm{mm}]$ & $\mathrm{dv}[\mathrm{mm}]$ & Roll [mrad] \\
\hline QF & 0.17 & 0.11 & 0.20 & 0.07 \\
\hline QD & 0.15 & 0.08 & 0.20 & 0.08 \\
\hline QFA & 0.14 & 0.08 & 0.19 & 0.08 \\
\hline B & 0.14 & 0.09 & 0.19 & 0.07 \\
\hline
\end{tabular}

Table 3

Final Local Alignment Errors

\begin{tabular}{|c|c|c|c|c|}
\hline & $\mathrm{dw}[\mathrm{mm}]$ & $\mathrm{du}[\mathrm{mm}]$ & $\mathrm{dv}[\mathrm{mm}]$ & Roll [mrad] \\
\hline $\mathrm{QF}$ & 0.13 & 0.03 & 0.04 & -0.06 \\
\hline $\mathrm{QD}$ & 0.10 & 0.04 & 0.02 & -0.05 \\
\hline $\mathrm{QFA}$ & 0.05 & 0.03 & 0.02 & -0.07 \\
\hline B & 0.08 & 0.03 & 0.02 & -0.04 \\
\hline
\end{tabular}

\section{ACKNOWLEDGMENTS}

T. Lauritzen is the iead engineer for the ALS survey and alignment effort, with responsibilities for all technical and personnel aspects, and it is a ploasure to acknowledge the friendly and efficient collaboration with him in this field over five years. Thanks are also due to the survey crews from LBI, led by W. Baldock and R. De Marco, and from SLAC, led by $M$. Gaydosh, for their accurate, professional work and many useful suggestions on how to improve procedures. Last, but not least, I want to thank R. Alvis who diligently processed most of the incoming raw data and created several sets of ideal data herself, and G. Krebs who took over my responsibilities in this ficld and contributed some data used in this paper.

\section{REFERENCES}

[1] R. Keller, T. Lauritzen, and H. Friedsam, $2^{\text {nd }}$ Int. Workshop on Accelerator Alignment (DF'Y Hamburg, 1990).

[2] 1-2 GeV Synchrotron Radiation Srurce, Conceptual Design Report, LBL Pub. 5172 Riv. (LBL Berkeley, 1986).

[3] Magnet fiducialization was performed by members of the ALS Mechanical Engineering group, guided by T. Lauritzen, J. Tanabe, and T. Henderson.

44] Magnetic measurements for these purposes were performed at L.BL Berkeley, guided by J. Tanabe and D. Nelson.

[5] H. Nishimura, private cumm. (LBL, Berkeley 1989). 

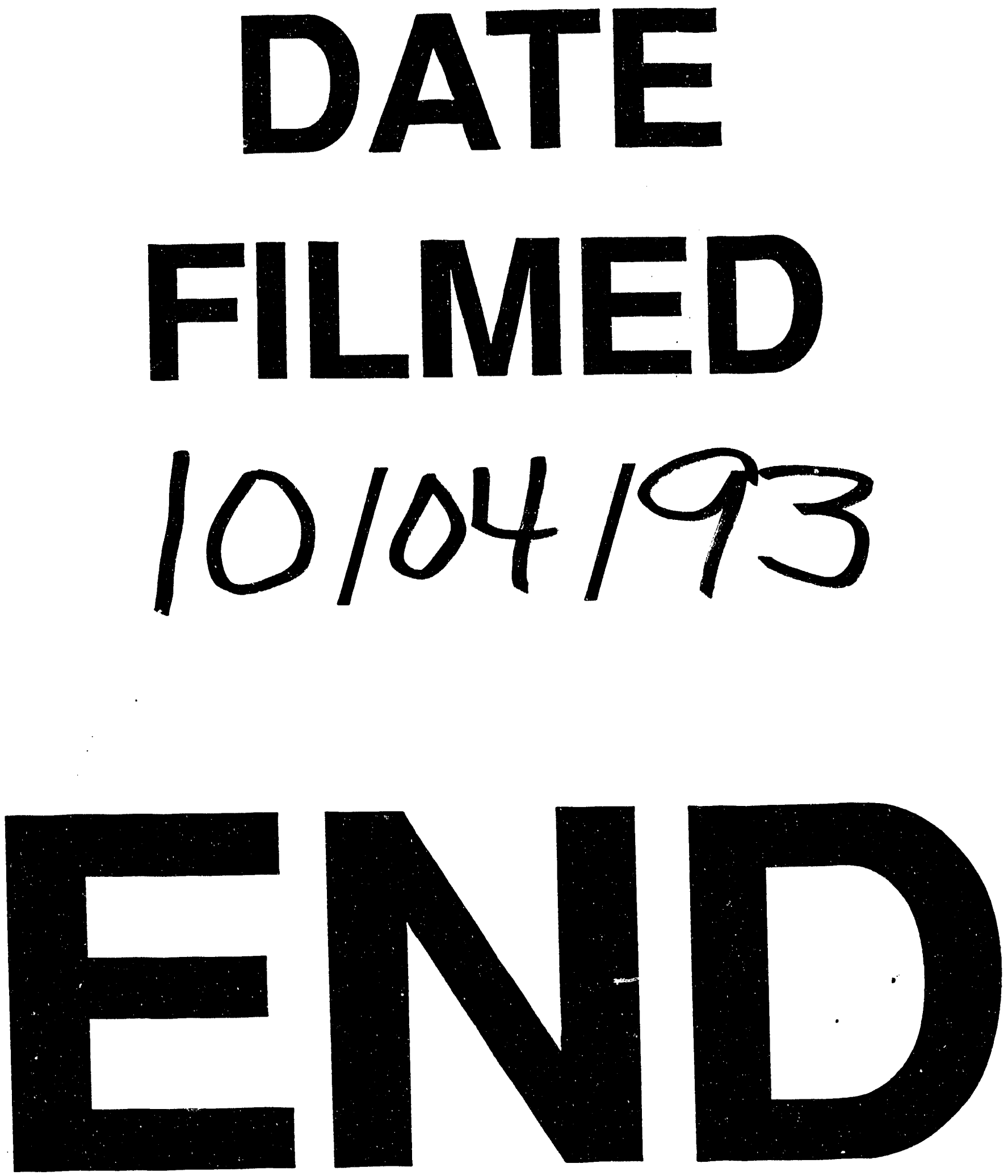
